\title{
eJRIEPS
}

Ejournal de la recherche sur l'intervention en éducation physique et sport

48 | 2021

Varia

\section{Analyse du déjà-là conceptuel d'inspecteurs pédagogiques d'éducation physique en Tunisie et en France}

Analysis of the conceptual already there of physical education inspectors in

Tunisia and France

Saïd Mohamed Aroui et Marie-France Carnus

\section{(Q) OpenEdition}

\section{Journals}

Édition électronique

URL : http://journals.openedition.org/ejrieps/5727

DOI : $10.4000 /$ ejrieps. 5727

ISSN : 2105-0821

Éditeur

ELLIADD

Référence électronique

Saïd Mohamed Aroui et Marie-France Carnus, « Analyse du déjà-là conceptuel d'inspecteurs pédagogiques d'éducation physique en Tunisie et en France », eJRIEPS [En ligne], 48 | 2021, mis en ligne le 12 janvier 2021, consulté le 02 février 2021. URL : http://journals.openedition.org/ejrieps/5727 ; DOI : https://doi.org/10.4000/ejrieps.5727

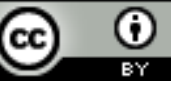

La revue eJRIEPS est mise à disposition selon les termes de la Creative Commons Attribution 4.0 International License. 


\title{
Analyse du déjà-là conceptuel d'inspecteurs pédagogiques d'éducation physique en Tunisie et en France
}

\author{
Saïd Mohamed Aroui * \& Marie-France Carnus ** \\ *UMR EFTS MA 122 (EDiC), Université Toulouse Jean Jaurès, France. \\ **SFR AEF, INSPE Toulouse Occitanie-Pyrénées, UT2J, France.
}

\section{Résumé}

Nous présentons ici une étude exploratoire réalisée dans le cadre d'une thèse portant sur l'analyse didactique clinique de pratiques d'inspection de quatre inspecteurs d'EPS dont une inspectrice et un inspecteur de chaque pays : la France et la Tunisie. Par le biais d'un questionnaire, où 30 inspecteurs en France et 77 inspecteurs en Tunisie ont répondu, nous mettons en évidence des tensions et des contradictions au sein d'un déjà-là conceptuel chez les inspecteurs dans les deux pays. Le métier d'inspecteur est tiraillé entre accompagnement et contrôle. Cette tension se manifeste dans leurs réponses qui renseignent sur leurs visions de leur travail d'inspecteur dans lequel l'évaluation joue un rôle central. A travers les spécificités culturelles, les résultats de cette étude préalable mettent en évidence une part de subjectivité chez les inspecteurs d'EPS en lien avec un assujettissement institutionnel qui oriente les conceptions de leur métier. Au-delà de la singularité des sujets interrogés, les résultats font ressortir des régularités de nature à éclairer de manière originale une pratique d'intervention souvent opaque et peu investiguée en recherche.

Mots clés : inspecteurs pédagogiques régionaux (IPR), éducation physique et sportive (EPS), didactique clinique, déjà-là conceptuel, accompagnement, contrôle, évaluation.

Analysis of the conceptual already there of physical education inspectors in Tunisia and France

\section{Summary}

We present here an exploratory study carried out as part of a thesis on the clinical didactic analysis of inspection practices of four PES (physical education and sport) inspectors, 


\section{eJRIEPS 48 Janvier 2021}

including one inspector from each country: France and Tunisia. By means of a questionnaire, to which 30 inspectors in France and 77 inspectors in Tunisia replied, we highlight tensions and contradictions within a conceptual already-there among inspectors in both countries. The job of inspector is torn between support and control. This tension manifests itself in their responses, which provide information on their visions of their work as inspectors, in which evaluation plays a central role. Through cultural specificities, the results of this preliminary study highlight a degree of subjectivity among PES inspectors in relation to an institutional subjection that guides the conceptions of their jobs. Beyond the singularity of the subjects questioned, the results highlight regularities that can shed original light on an intervention practice that is often opaque and little investigated in research.

Key words: regional pedagogical inspectors (IPR), physical education and sport (PES), clinical didactics, already there conceptual, accompaniment, control, evaluation.

\section{Introduction}

Souvent décriées mais pourtant incontournables, les pratiques d'évaluation dans le monde professionnel sont nombreuses et diverses (Paquay et al., 2010). Dans les métiers de l'enseignement, de l'éducation et de la formation, ces pratiques sont assujetties à des normes institutionnelles plus ou moins explicites et parfois prescrites dans des référentiels de compétences qui définissent «les objectifs et la culture commune à tous les professionnels » du champ (BO du 25-07-2013).

Pour contrôler, garantir et optimiser la conformité de cette pratique avec ces référents à la fois denses et opaques, "l'État délègue à un fonctionnaire réputé compétent le droit de regard qu'elle prétend avoir sur l'institution chargée de l'Éducation » (Vacher, 1986). Dans le domaine de l'EPS, l'enseignant est contrôlé par l'institution de tutelle de manière régulière et c'est l'inspecteur pédagogique régional (IPR) qui est chargé de cette mission. L'inspection est un processus qui commence dès la désignation de l'enseignant à inspecter et prend fin lorsque ce dernier signe le rapport d'inspection rédigé par l'IPR. Entre ces deux moments se situe l'inspection proprement dite où l'inspecteur assiste à une leçon in situ et procède à un entretien d'inspection.

Selon Albanel (2009), l'inspecteur a pour mission de contrôler la conformité des enseignements aux attendus institutionnels et de mettre en place, en même temps, un accompagnement pédagogique en direction du professeur. Cette double mission, à l'origine de l'inspection, tiraille l'inspecteur entre deux postures, celle du contrôleur ou du juge et 


\section{eJRIEPS 48 Janvier 2021}

celle de l'accompagnateur ou du conseiller. Selon Jorro (2006), l'acte évaluatif est un acte composite où l'évaluateur s'engage avec une intention particulière. Cette intention découle d'un système de valeurs dans lequel l'acte évaluatif n'est ni neutre ni objectif. L'évaluation des enseignants d'EPS est en même temps dépendante des conceptions relatives à l'inspection qui peuvent fluctuer entre le contrôle et l'accompagnement et de celles de l'EPS qui s'organisent autour des trois objectifs de la discipline : culturaliste, développementaliste et intégrateur (ou citoyen) (Hébrard, 1986). Jorro (2009) parle de filtres identitaires de l'activité évaluative. Ces tensions institutionnelles, consubstantielles de la fonction, divisent le sujet inspecteur et nous conduisent à convoquer le champ de la didactique clinique où Carnus (2001) parle d'un déjà-là décisionnel qui fournit, à moments donnés, des motifs et mobiles possibles dans le cadre de son activité professionnelle. Une des trois composantes de ce déjà-là est le déjà-là conceptuel qui est construit par le sujet au cours de son histoire et sa trajectoire personnelles et professionnelles.

Dans cet article, nous rendons compte d'un déjà-là conceptuel chez les IPR d'EPS, en Tunisie et en France en tentant de les positionner entre accompagnement et contrôle. Nous cherchons aussi à explorer certains effets éventuels de la culture et des spécificités institutionnelles sur ces déjà-là conceptuels dans les deux pays. Cet article s'appuie sur l'étude exploratoire d'un travail de thèse portant sur l'analyse didactique clinique des pratiques d'inspection en EPS (Aroui, 2021). Notre projet à terme est de décrire et de comprendre, chez quatre inspecteurs, une femme et un homme de chaque pays, l'effet de leur déjà-là sur leurs pratiques d'inspection.

\section{L'inspection pédagogique en Tunisie}

En Tunisie, les inspecteurs d'éducation physique et des activités sportives de l'enseignement préparatoire et secondaire sont sous la tutelle du ministère des affaires de la jeunesse et du sport (MAJS) et relèvent structurellement de l'administration centrale de l'inspection. Les activités pédagogiques des inspecteurs sont réalisées en coordination avec les services des commissariats régionaux. Chaque inspecteur a sa circonscription. On y distingue quatre grades: inspecteur pédagogique régional (IPR), inspecteur principal, inspecteur général, inspecteur général-expert.

Les IPR EPS sont recrutés par concours externe, sur épreuves, ouverts aux enseignants d'éducation physique titulaires, exerçant dans les écoles préparatoires et les lycées ayant un doctorat, un master, une maitrise ou une licence (ou titres ou diplômes admis en 


\section{eJRIEPS 48 Janvier 2021}

équivalence) et justifiant d'au moins cinq ans d'ancienneté dans le grade et d'au moins huit ans d'ancienneté dans l'enseignement.

Le concours est composé de deux épreuves : une épreuve écrite d'admissibilité et une épreuve pratique d'admission. L'épreuve écrite, avec note éliminatoire, concerne le domaine de la didactique des APS, de la pédagogie de l'EPS et tout ce qui est en relation avec l'enseignement de l'éducation physique. Les admissibles passent l'épreuve pratique qui consiste à enseigner l'éducation physique dans une classe puis à s'entretenir avec un jury composé de trois inspecteurs de grades supérieurs. Ceux qui réussissent seront des élèves inspecteurs et bénéficieront d'une formation de 24 mois.

La formation combine théorie et pratique et vise l'acquisition des compétences nécessaires pour exercer le métier d'inspecteur en EPS. La formation théorique est centralisée et touche plusieurs domaines en relation tels que la didactique des APS et de l'EPS, la législation, I'informatique, etc. La formation théorique obéit au principe du contrôle continu. La formation pratique avec un tuteur est décentralisée et est confiée aux inspecteurs dans les régions et concerne tous les actes de l'inspecteur: l'inspection des enseignants, les visites d'établissements, les visites de l'association sportive scolaire, etc.

A la fin de la formation, les élèves inspecteurs passent un examen qui comprend deux épreuves : l'inspection d'un enseignant et la présentation d'un projet de recherche dans le domaine de la didactique de l'EPS. Les élèves inspecteurs ayant obtenu la moyenne dans toutes les épreuves confondues de la formation sont nommés inspecteurs stagiaires durant un an pour être ensuite titularisés grâce à un avis favorable du directeur national de l'inspection pédagogique.

Plusieurs missions sont attribuées aux inspecteurs selon leurs grades: l'évaluation, la formation et l'innovation. Les inspecteurs sont chargés d'évaluer les approches pédagogiques, les programmes, l'impact de la formation, les projets, les recherches éducatives, les études sur terrain, (...) et surtout le travail des enseignants dans les établissements éducatifs publics et privés.

Chaque deux ans, l'enseignant d'EPS a droit à une évaluation lors d'une inspection individuelle à l'improviste qui se déroule en deux étapes : l'observation de la leçon suivie d'un entretien. Avant l'observation de la leçon, l'inspecteur met le proviseur au courant du nom de l'enseignant à inspecter puis tous deux se rendent ensemble sur le lieu de travail. Le proviseur n'est pas dans l'obligation d'assister au déroulement de la leçon. Après avoir pris contact avec l'enseignant et lui avoir expliqué l'objectif de la visite, l'inspecteur récupère les documents présentés et prend place pour observer. L'évaluation du travail ne se réfère 


\section{eJRIEPS 48 Janvier 2021}

pas à un référentiel de compétence ou à une grille nationale mais aux conceptions que l'inspecteur se fait des «bonnes pratiques». Une fois la leçon terminée, l'entretien se déroule dans le bureau du proviseur en sa présence. Après l'inspection et dans un délai qui ne dépasse pas deux semaines, l'inspecteur doit envoyer à l'enseignant par voie hiérarchique un rapport d'inspection avec la note accordée. Cette note dite « pédagogique » joue un rôle important dans la promotion de l'enseignant ainsi que dans ses demandes de mutations vers d'autres établissements. La note attribuée doit respecter une échelle nationale.

\section{L’inspection pédagogique en France}

Depuis 1981, l'EPS est sous la tutelle de Ministère de l'Éducation Nationale (MEN). Les personnels d'inspection sont les cadres supérieurs de l'EPS. Dans le second degré, ils se répartissent en deux catégories : les inspecteurs généraux de l'éducation nationale (IGEN) et les inspecteurs d'académie - inspecteurs pédagogiques régionaux (IA-IPR). Ces derniers fonctionnent dans le cadre du programme de travail de l'académie. Les IA-IPR EPS sont recrutés, par concours ou par détachement et intégration directe. Pour ce qui concerne les concours, il en existe deux types: concours sur épreuves et par spécialité pour les fonctionnaires titulaires (enseignant-chercheur, professeur agrégé, etc.) et un concours sur titres qui concerne les candidats titulaires d'une licence justifiant de huit années d'expérience professionnelle dans les domaines soit de l'éducation, de l'enseignement ou de la formation. Pour ce qui concerne le détachement ou l'intégration directe dans le corps des IA-IPR, il est notamment ouvert aux personnels de direction d'établissement d'enseignement, aux professeurs des universités, aux maîtres de conférences, aux professeurs agrégés et aux inspecteurs de l'Éducation Nationale (Décret n 90-675 du 18 juillet 1990 https://www.education.gouv.fr/inspecteur-d-academie-inspecteur-pedagogiqueregional-ia-ipr-2666) .

L'épreuve du concours comporte deux temps. Une épreuve d'admissibilité (coefficient 1) qui consiste en une étude par le jury d'un dossier de reconnaissance des acquis de l'expérience professionnelle établi par le candidat. Le jury examine le dossier qu'il note de 0 à 20 (cette épreuve fait l'objet d'une double correction) et dresse la liste des candidats autorisés à se présenter à l'épreuve d'admission. Cette épreuve orale (coefficient 3) consiste en un entretien d'une durée de 45 minutes qui débute par un exposé du candidat sur son parcours et son activité professionnelle. Cette étape doit permettre au jury de repérer et 


\section{eJRIEPS 48 Janvier 2021}

d'apprécier les compétences du candidat. Après l'exposé, l'entretien se décompose en deux parties : une première partie, où les questions du jury concernent la discipline EPS alors qu'au cours de la seconde, l'interrogation porte sur le système éducatif en général. Cet oral donne lieu à l'attribution d'une note de 0 à 20. Le jury dresse, au vu de la somme des notes des deux épreuves, la liste des candidats déclarés admis et la liste complémentaire. Les lauréats, d'après le décret du 12 janvier 2010, entrent de plain-pied dans le corps des IAIPR en tant que stagiaire.

Après leur recrutement, les inspecteurs suivent une formation afin de se préparer au milieu professionnel et construire les compétences nécessaires. II s'agit d'une formation initiale de 65 jours sur 24 mois qui se déroule à l'Institut des Hautes Études de l'Éducation et de la Formation (IH2ÉF à Poitiers). La formation dans les 12 premiers mois avant la titularisation est structurée par trois modules qui visent les compétences suivantes : être personnel d'encadrement, piloter, évaluer et animer pédagogiquement des équipes, et innover. Pour les 12 mois qui suivent la titularisation, deux modules visent les mêmes compétences et cherchent à apporter des réponses adaptées sur des problématiques scolaires vives comme la contribution à la réussite de l'élève, les notions de partenariats et de territoires, le numérique, etc. A l'issue du stage de formation, les intéressés sont titularisés après avis de la commission administrative paritaire nationale, au vu d'un rapport établi par le directeur du centre de formation, d'un rapport de stage établi par le Recteur d'Académie concerné ainsi que d'un rapport établi par le doyen de l'inspection générale de l'éducation, du sport et de la recherche (IGÉSR). Cette formation professionnelle statutaire est complétée, au cours des 3 années qui suivent la titularisation, par une formation complémentaire de 5 jours dans le cadre du plan national de formation, soit dans le cadre des plans académiques de formation ou des offres de service public nationales voire internationales.

Les inspecteurs sont chargés, tout d'abord, de veiller à la mise en œuvre de la politique éducative dans les établissements scolaires. Ils sont aussi responsables d'évaluer le travail des personnels enseignants, les orientations des établissements du second degré ainsi que les résultats de la politique éducative. En outre, ils inspectent les personnels enseignants auxquels ils apportent des conseils et s'assurent du respect des objectifs et des programmes nationaux. Parmi les missions des IA-IPR, s'ajoutent la participation à l'animation pédagogique dans les formations initiales et continues et le conseil auprès des chefs d'établissement. Des dossiers particuliers peuvent être confiés par le Recteur d'Académie à l'inspecteur comme l'orientation des élèves, les examens, la gestion des personnels éducatifs, etc. 


\section{eJRIEPS 48 Janvier 2021}

En France, depuis 2017, le processus d'inspection a changé. La réforme comprend un accompagnement tout au long du parcours avec des rendez-vous de carrière. II s'agit du PPCR (Parcours Professionnels, des Carrières et des Rémunérations). II permet d'aménager un temps dédié à chaque enseignant pour porter un regard sur une période professionnelle (en moyenne tous les 7 ans), à des moments où il semble pertinent de faire le point. Il s'agit d'un temps d'échanges sur les compétences acquises et sur les perspectives d'évolution professionnelle. Ce qu'il faut retenir est l'affirmation du principe d'accompagnement continu tout au long de la carrière. Cette réforme marque aussi la fin de la notation et des trois cadences d'avancement (grand choix, choix, ancienneté). L'enseignant est informé de la programmation d'un rendez-vous de carrière avant le début des vacances d'été. Au plus tard quinze jours avant la date du rendez-vous de carrière, le calendrier est communiqué à l'enseignant. II bénéficie d'une inspection en classe, d'un entretien avec l'inspecteur et pour le second degré, d'un deuxième entretien avec le chef d'établissement. L'évaluation du travail se réfère à un référentiel de compétence publié au bulletin officiel du 25 juillet 2013 (annexes 2). Après le rendez-vous, l'enseignant est informé des appréciations des évaluateurs ainsi que de l'appréciation finale du rendez-vous de carrière par notification dans une application (e-prof) et dans sa messagerie professionnelle. Le tableau I placé dans les annexes 1 synthétise les principaux éléments de comparaison entre la Tunisie et la France en matière de parcours de formation, de fonctions et de missions des inspecteurs d'EPS. Le tableau II regroupe les principaux éléments du processus d'inspection dans les deux pays.

Ainsi, pour devenir inspecteur dans les deux pays, il faut se présenter à un concours et suivre une formation continue pour être titularisé. En France et en Tunisie les inspecteurs ont presque les mêmes missions. Contrairement à la France, il n'y a pas en Tunisie de référentiel de compétences pour les enseignants. De fait, en Tunisie, l'inspecteur est institutionnellement davantage positionné du côté de l'évaluateur-contrôleur avec une visite à l'improviste qui donne lieu à une note pédagogique. En France, l'institution place l'inspecteur davantage en position d'évaluateur - accompagnateur : la visite est annoncée et débouche sur une appréciation. Dans les deux pays, un rapport d'inspection alimente le dossier de carrière de l'enseignant.

\section{L'inspection entre accompagnement et contrôle : différents paradigmes}

En Tunisie comme en France et depuis sa création, l'inspection pédagogique est en tension entre contrôle et accompagnement. L'inspecteur est institutionnellement divisé entre un rôle 


\section{eJRIEPS 48 Janvier 2021}

d'évaluateur-contrôleur et un rôle d'accompagnateur-conseiller. II est par ailleurs juge et partie car il contribue à la formation des enseignants en même temps qu'il participe à leur évaluation. L'évaluation des professionnels prend de plus en plus d'ampleur dans le domaine de l'éducation et de formation. Plusieurs chercheurs ont étudié ce processus qui apparait malgré tout opaque.

Jorro (2006) distingue, en éducation, quatre postures de l'évaluateur associée chacune à un imaginaire. La posture de contrôleur est associée à l'imaginaire de la performance qui valorise l'exemplarité de l'action et sa conformité avec les normes. Dans cet imaginaire la norme est garante de l'objectivité de l'évaluation. Si ce contrôle normatif est dénoncé sous prétexte que les dimensions pédagogiques demandent des aménagements particuliers, la posture de contrôleur glisse, alors, vers celle du juge. La posture d'expert est associée à l'imaginaire de la maitrise qui valorise l'efficacité de l'action. Dans cet imaginaire, l'évaluateur joue le rôle du coach qui favorise la progression et la réussite. Du point de vue institutionnel, l'évaluateur cherche à réduire les décalages entre le prévu et le réalisé pour s'approcher des référentiels implicites ou explicites visés. Quant à la posture de conseiller, elle est associée à l'imaginaire de la construction. L'évaluateur est à l'écoute de l'apprenant, l'accompagne et le considère comme acteur impliqué dans le développement de ses propres compétences. II favorise, alors, son activité, son autonomie et encourage ses initiatives. En situation institutionnelle, le conseiller (ou l'expert-conseil) veille à comprendre les pratiques professionnelles pour les développer à travers un processus de régulation basé sur le dialogue entre l'évaluateur et l'évalué autour d'un référent qui peut être explicite ou implicite. Enfin, la posture de consultant associée à l'imaginaire de la compréhension ne valorise ni l'exemplarité de l'action, ni son efficacité, ni la construction de compétences mais une approche singulière de l'apprenant afin de comprendre la situation du sujet en formation et lui apporter les régulations adéquates.

Pour l'inspection, Sénore (1999) distingue en premier lieu deux paradigmes qui fondent cette forme d'évaluation : le paradigme charismatique et le paradigme techniciste. II en propose ensuite un troisième, le paradigme déontologique, encore à construire. Ces paradigmes ne sont pas la réalité, mais ils représentent, néanmoins, des modèles dominants dans les attitudes et les comportements des inspecteurs. Le paradigme charismatique est le fondateur de l'acte d'inspection. L'évaluation du travail de l'enseignant est régie par l'arbitraire, « le bon », le « souhaitable » ou « l'agréable » selon le seul désir de l'inspecteur. Le paradigme charismatique nous rappelle la posture de contrôleur et de juge évoqué par Jorro (2006). C'est le cas du modèle institutionnel tunisien où il n'y a pas 


\section{eJRIEPS 48 Janvier 2021}

de référentiel officiel. Le paradigme techniciste, inspiré des sciences de gestion, cherche à rompre avec l'inspection des personnes et vise l'instauration d'une évaluation rigoureuse et transparente des compétences professionnelles des enseignants en se référant à des critères observables. L'inspection fonde, alors, sa légitimité sur l'utilisation d'outils techniques (référentiel de compétences, grille d'observation, etc.). Parce qu'il permet la validation d'un niveau de compétence, le paradigme techniciste nous rappelle la posture du contrôleur (Jorro, 2006). C'est plutôt le cas du modèle institutionnel français.

Pour dépasser les limites de ces paradigmes à des fins praxéologiques et pour éviter que chacun se réfère à sa seule morale personnelle, Sénore (2000, p.191) propose de fonder l'inspection sur un code déontologique s'appuyant sur des valeurs éthiques afin d'atténuer l'effet de la dissymétrie institutionnelle et transformer " le pacte » en « alliance » et « le face à face dangereusement mortifère » en " côte à côte fertile ». Pour Sénore (1999), l'un des rôles les plus importants que peut jouer l'inspecteur est d'aider l'enseignant à devenir vraiment un acteur didactique et pédagogique. Pour cela, il devrait l'encourager, et diffuser ses pratiques innovantes en favorisant les échanges dans la communauté. L'inspecteur pourrait chercher à le convaincre sans jamais le contraindre en se confrontant à ses problèmes et en s'identifiant à lui devant des élèves en difficulté. Dans son travail d'évaluation, l'inspecteur devrait chercher à être le plus transparent possible tout en ayant des relations humaines avec les enseignants. Sénore (2000) conçoit qu'un référentiel de compétences explicité aux enseignants serait de nature à guider les observations de l'inspecteur et enrichir le dialogue entre les deux protagonistes. Enfin l'inspecteur doit être reconnu par les enseignants comme un accompagnateur d'équipe dans une démarche de " côte à côte ». Le paradigme déontologique nous rappelle la posture du conseiller ou l'expert-conseil (Jorro, 2006).

\section{Le déjà-là conceptuel}

Selon Giordan et De Vecchi (1987), la notion de conceptions introduite dans le champ de la didactique est un élément moteur entrant dans la construction des savoirs. Ces auteurs ajoutent que la conception est un ensemble d'idées cohérentes correspondant à une structure mentale sous-jacente responsable de manifestations contextuelles permettant de donner du sens aux choses (faits, dires, objets, etc.). Elle fait appel au cognitif, n'est observable que par ses conséquences sur les actes du sujet (dire, faire) et se construit dans le temps en relation avec un ou plusieurs savoirs. De plus, les conceptions renvoient à un ensemble d'idées que l'apprenant mobilise pour raisonner face à des situations 


\section{eJRIEPS 48 Janvier 2021}

problématiques. Dans le champ de la didactique clinique nous convoquons la notion de déjà-là conceptuel. Elle fait partie du déjà-là décisionnel produit de l'histoire du sujet qui influence les enjeux de savoirs et leur devenir dans le processus de transmission appropriation. Au carrefour de l'intime et du public, le déjà-là décisionnel est un des déterminants de la décision du sujet (Carnus, 2001 ; Loizon, Carnus, 2012). Il est composé de trois instances en tension : le déjà-là conceptuel (pôle cognitif), le déjà-là intentionnel (pôle conatif) et le déjà-là expérientiel (pôle expérientiel) (Carnus, 2001). Le déjà-là conceptuel est construit par le sujet à travers son histoire personnelle et professionnelle. II est constitué par l'ensemble des conceptions, croyances et valeurs du sujet et par conséquent propre à chaque sujet.

Selon Carnus (2002), le déjà-là conceptuel enraciné dans la sphère privée est influencé par la sphère publique dont certains aspects institutionnels (notamment les missions et les programmes) et socioculturels. Sa fonction essentielle est de donner du sens aux éléments constitutifs de la pratique professionnelle - ici la pratique d'inspection.

\section{Méthodologie}

Selon Van Der Maren (1995), la recherche inductive ou exploratoire est « la recherche par laquelle on souhaite trouver des choses plutôt que de prouver des choses. C'est le type de recherche qui semble le plus utile, à l'heure actuelle du moins, en éducation » (p.13).

Engagé dans une théorie du sujet pris dans le didactique (Carnus, 2009), pour analyser les pratiques d'inspection, nous utilisons comme moyen d'investigation la réalisation d'une enquête exploratoire auprès des inspecteurs d'EPS. Selon De Ketele et Roegiers (2016), le terme d'enquête désigne l'étude d'un thème précis auprès d'une population dont on détermine un échantillon afin de préciser certains paramètres. Vu le nombre réduit des deux populations nous n'avons pas déterminé un échantillon, l'objectif étant de collecter le maximum de réponses. Pour ce genre d'étude et pour toucher le maximum d'inspecteurs, nous choisissons le questionnaire comme technique de recueil de données. Auto-administré et rapide, il peut être diffusé à un très large échantillon de participants et permet de recevoir des réponses en quelques jours.

L'enjeu de notre questionnaire est de recueillir certains invariants du déjà-là d'inspecteurs d'EPS tunisiens et français. Notre intention est de dégager des tendances au cœur d'un déjà-là conceptuel "générique " qui sera ensuite spécifié de manière singulière pour chaque sujet observé et de repérer des régularités et des différences entre les inspecteurs tunisiens et français. En plus, ce questionnaire va nous aider à positionner les inspecteurs 


\section{eJRIEPS 48 Janvier 2021}

qui ont accepté de collaborer avec nous. Le questionnaire est aussi une occasion de mieux connaitre le contexte général de l'inspection et du métier d'inspecteur ainsi que les caractéristiques institutionnelles et culturelles qui l'influencent dans les deux pays.

Néanmoins, le plus grand défi d'un questionnaire exploratoire est sa formalisation, pour qu'il puisse cibler les déjà-là conceptuels des inspecteurs et permette le recueil de données fiables et exploitables. Dans ce questionnaire, 37 questions ouvertes, fermées ou mixtes, gravitent autour trois déjà-là (expérientiel, conceptuel, intentionnel). Dans cet article, nous nous intéressons aux questions concernant le déjà-là conceptuel.

Avant d'être mis en ligne, le questionnaire a été envoyé à deux inspecteurs de chaque pays afin de nous assurer que les questions étaient compréhensibles et univoques et pour vérifier le temps alloué pour répondre que nous avions estimé à environ 45 minutes. Cette étape nous a permis de remanier le questionnaire en fonction des feedback obtenus. Le questionnaire a été ensuite lancé en ligne à l'aide du logiciel "Lime Survey » à destination de la totalité des inspecteurs d'EPS recensés à l'échelle nationale (150 en Tunisie et 91 en France) parmi lesquels figurent les quatre professionnels qui ont accepté de collaborer avec nous pour la suite de notre recherche. Au final, 77 tunisiens et 30 français ont répondu.

\section{Quelques résultats}

Nous sélectionnons certains résultats qui nous apparaissent les plus significatifs au regard de notre questionnement. Nous analysons pour cela les réponses à sept questions qui nous paraissent pertinentes par rapport aux régularités et aux différences interculturelles.

\subsection{Question 1: "pensez-vous qu'il soit pertinent d'annoncer la date de} l'inspection? Quelle que soit votre réponse, merci d'argumenter ».

Du fait de la différence institutionnelle entre la Tunisie et la France, il parait important de savoir comment les inspecteurs évaluent la pertinence d'annoncer la date afin de voir si la prescription de l'institution va dans le sens des conceptions des inspecteurs. II s'agit d'une question fermée qui amène à trois types de réponse, OUI, NON ou autre éventuellement. Pour les inspecteurs français, comme pour ceux tunisiens, les arguments qui concernent la réponse OUI s'organisent en deux catégories : « accompagnement » et « ne pas piéger » qui évoquent le paradigme du conseiller.

Les arguments de la réponse NON renvoient à deux catégories qui sont : «leçon non répétée »; « effet surprise ». 


\section{eJRIEPS 48 Janvier 2021}

Pour la réponse "autres », elle renvoie aux réponses que nous n'avons pas pu classer dans les catégories précédentes et qui restent très minoritaires surtout chez les tunisiens. Pour les inspecteurs français, les arguments de cette réponse s'organisent en deux catégories : « la question n'est pas là » et « ni oui, ni non ».

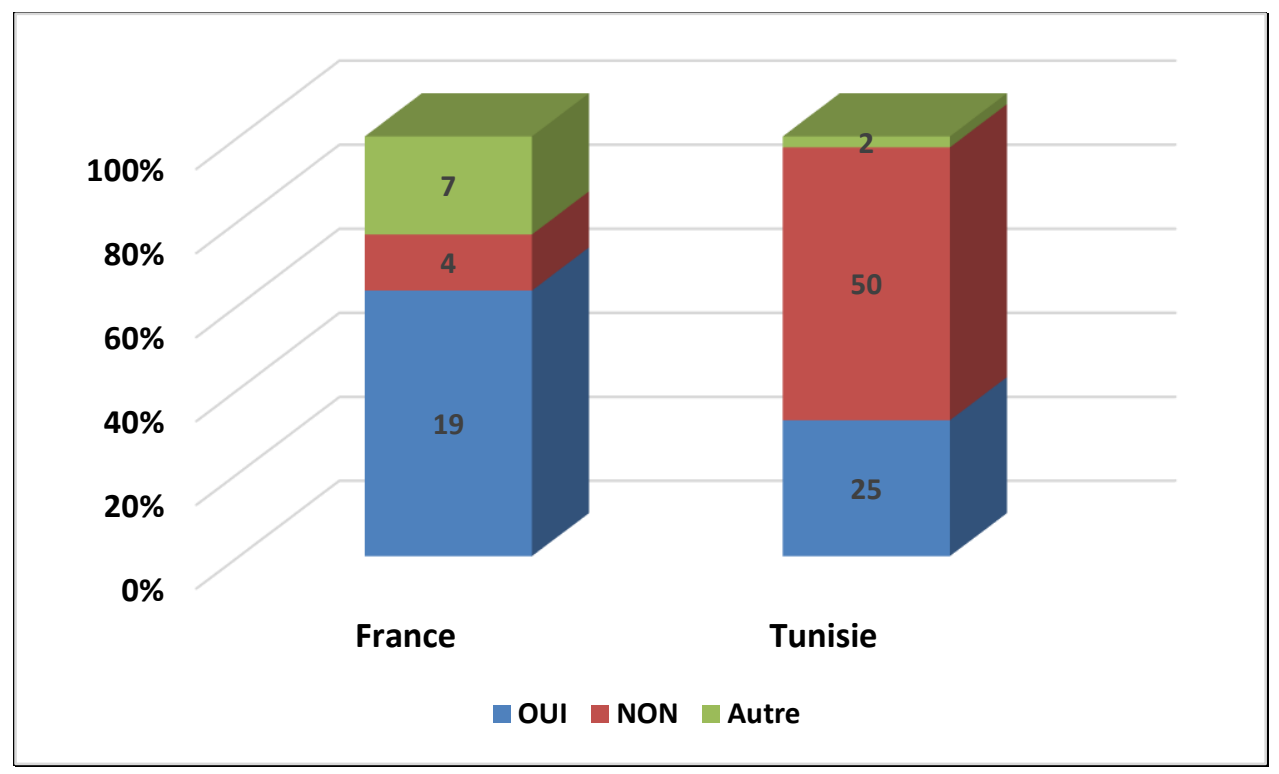

Figure 1: distribution des réponses à la question 1

Pour 19 inspecteurs (63\%) ayant répondu en France et 25 inspecteurs (32\%) ayant répondu au questionnaire en Tunisie, il semble pertinent d'annoncer la date d'inspection aux enseignants. Ils argumentent qu'il s'agit d'un accompagnement dont le but n'est pas de piéger l'enseignant. Cette conception évoque la posture du conseiller telle que développée dans les travaux de Jorro (2006). Cette réponse marque une forme d'assujettissement des inspecteurs français par rapport à l'institution.

Pour 4 inspecteurs (13.33\%) ayant répondu en France et 50 inspecteurs (65\%) ayant répondu au questionnaire en Tunisie, il n'est pas pertinent d'annoncer la date d'inspection aux enseignants. Ils argumentent qu'il s'agit d'un changement d'attitude et que l'effet surprise fait partie de l'inspection. Cette conception à propos de la date d'inspection se rapproche de l'imaginaire de la performance et de la conformité à des normes contribuant à la construction de la posture du l'inspecteur contrôleur (Jorro, 2009). 7 inspecteurs ont répondu par « autres ». Ils pensent que la question la plus importante pour l'inspection n'est pas là.

Ainsi, les inspecteurs tunisiens et français font preuve d'une forme d'assujettissement par rapport à leurs institutions qui semblent avoir influencé leur déjà-là conceptuel. Au vu de ces premiers résultats, la tension entre mission de contrôle et mission d'accompagnement semble plus forte chez les inspecteurs tunisiens $(32 \%$ d'entre eux ayant répondu OUI à 


\section{eJRIEPS 48 Janvier 2021}

cette question), les inspecteurs français affichant une certaine homogénéité du côté de l'accompagnement.

\subsection{Question 2: " s'il n'y a plus de note, pensez-vous que l'inspection perdra} de sa légitimité au sein de l'institution? Quelle que soit votre réponse merci d'argumenter. "

Contrairement à leurs homologues tunisiens, suite à la réforme du PPCR, les inspecteurs français n'attribuent plus de note. Du fait de ce changement récent, il apparait intéressant de connaitre l'importance qu'attribuent les inspecteurs à la note dans les deux pays et de repérer s'ils sont en phase ou en décalage avec les préconisations institutionnelles. II s'agit d'une question fermée qui amène là-encore trois types de réponse, OUI, NON ou autre éventuellement.

Pour les inspecteurs français, les arguments de la réponse NON renvoient à deux catégories : « accompagnement » et « autre appréciation ». Les rares arguments de leurs homologues tunisiens n'ont pas fait l'objet d'une analyse de contenu. Pour les inspecteurs tunisiens, les arguments de la réponse OUI renvoient à deux catégories « la note est importante pour l'enseignant» et «la note est importante pour l'inspecteur ». Les arguments des inspecteurs français en faveur du OUI renvoient à une seule catégorie " changement dû au PPCR ». Les rares arguments pour la réponse « autres », renvoient à la recherche d'une autre approche plus globale.

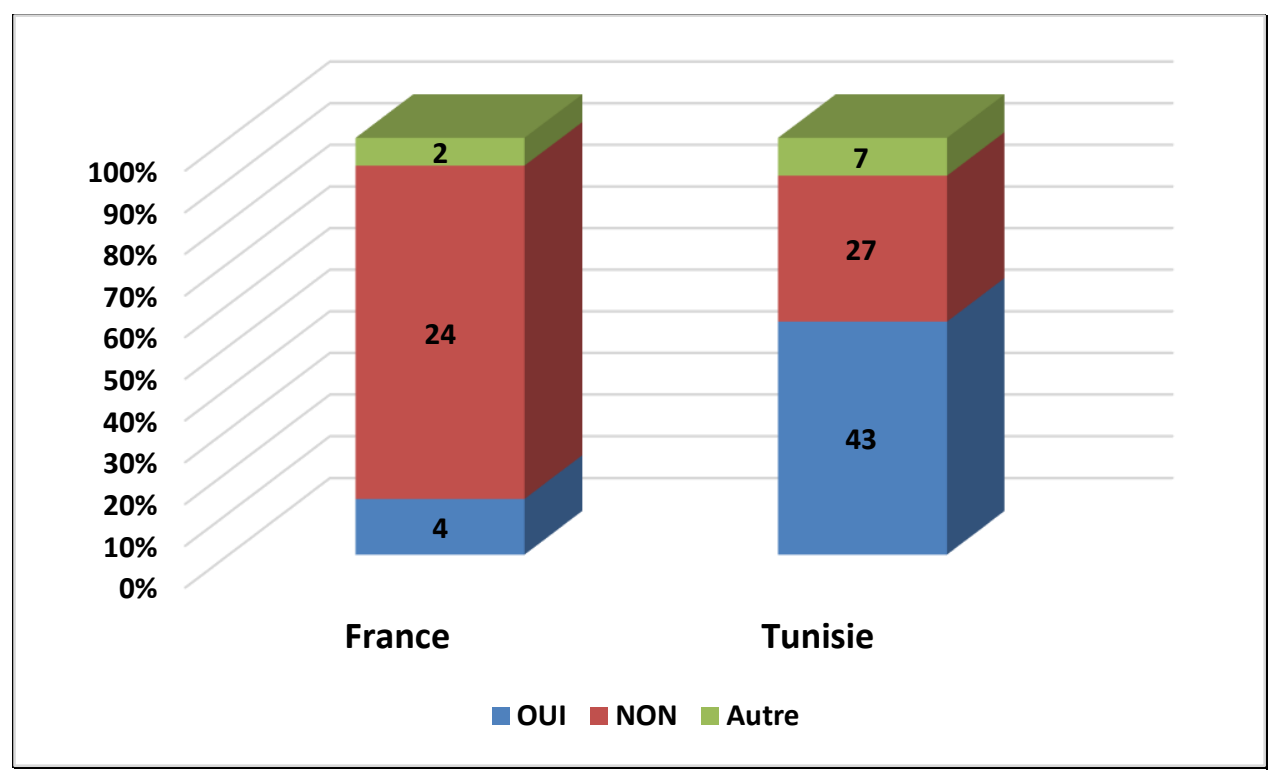

Figure 2: distribution des réponses à la question 2 


\section{eJRIEPS 48 Janvier 2021}

Parmi les inspecteurs français ayant répondu, 4 (15\%) pensent que s'il n'y a plus de note, l'inspection perd sa légitimité. On peut en conclure que ces quatre professionnels ne se sentent plus légitimes ce qui est de nature à engendrer un certain inconfort. En Tunisie, 43 inspecteurs (55\%) ont répondu OUI en argumentant soit que les enseignants aiment les notes soit qu'il est difficile à l'inspecteur de quantifier la compétence de l'enseignant sans la note. Pour ces inspecteurs, le contrôle par la note assoit leur légitimité.

Du côté du NON, nous comptabilisons 24 réponses (80\%) chez les inspecteurs français. Ils justifient leur réponse en écrivant qu'il vaut mieux accompagner les enseignants en changeant les notes par des appréciations. Ils ajoutent que le PPCR va tout changer et que les inspecteurs vont devenir des « super formateurs ». II est intéressant de remarquer que 27 inspecteurs tunisiens (35\%) pensent aussi que la suppression de la note ne toucherait pas à leur légitimité et argumentent leur réponse de la même manière que les inspecteurs français.

Ainsi, si les inspecteurs français et tunisiens vont globalement dans le même sens que leurs institutions, la tendance est beaucoup plus contrastée en Tunisie ou plus d'un tiers des répondants semblent remettre en question la pertinence de la note. II semble également que les français sont en accord avec la suppression de la note induite par la toute récente réforme du PPCR et se positionnent davantage sur ce point dans le paradigme d'accompagnateur. Là encore, les inspecteurs tunisiens et français font globalement preuve d'une forme d'assujettissement par rapport à leurs institutions qui dicte leur pratique en matière de notation ou d'appréciation.

\subsection{Question 3 : « construisez-vous des grilles d'évaluation? Si oui, quels sont} vos principaux critères et indicateurs? Si non, Pourquoi? »

En France, les compétences qu'un enseignant d'EPS doit maitriser sont détaillées dans un référentiel. (B.O. du 25 juillet 2013 en annexes 2). En Tunisie, ce n'est pas le cas. Du fait de cette différence institutionnelle entre les deux pays, il parait important de voir comment les inspecteurs construisent leurs grilles d'évaluation qui émanent d'un référentiel qu'il soit explicite ou implicite (Figari et al., 2014). II s'agit là-encore d'une question fermée qui amène à deux types de réponse, OUI ou NON puis à formuler des arguments pour préciser la réponse.

Pour les arguments de la réponse OUI, les inspecteurs français évoquent deux catégories : « grille nationale » et «éférentiel de compétence ». Pour les inspecteurs tunisiens ils évoquent plusieurs catégories ou critères : « organisation », « conception », « régulation », 


\section{eJRIEPS 48 Janvier 2021}

«communication». Pour ce qui concerne les arguments de la réponse négative, les inspecteurs tunisiens évoquent deux catégories « expert supposé savoir » et «n'est pas exhaustive ».

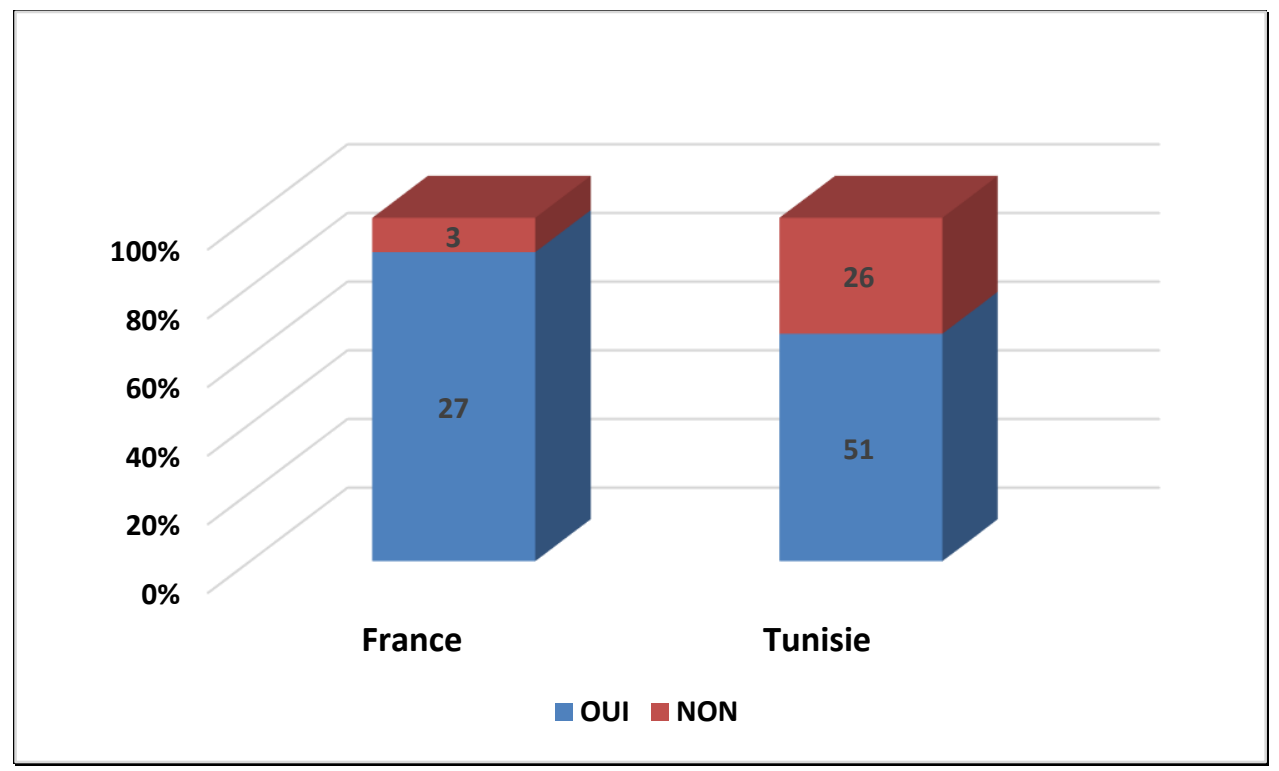

Figure 3: distribution des réponses à la question 3

Parmi les inspecteurs ayant répondu à cette question en France, 27 (90\%) affirment qu'ils construisent des grilles d'évaluation. Ils argumentent que ces grilles sont élaborées à partir des référentiels de compétences et des grilles nationales. En Tunisie, 51 inspecteurs (67\%) construisent des grilles d'évaluation pour pallier l'absence d'un référentiel de compétences pour les enseignants tunisiens. II semblerait ici que ce n'est pas l'assujettissement à l'institution qui les pousse à construire une grille mais une conception proche de l'évaluateur - contrôleur évoquée par Jorro (2006). Les principaux critères ou indicateurs de ces grilles sont regroupés à posteriori à partir des réponses des inspecteurs. Parmi ces critères : l'organisation, les régulations, l'attitude de l'enseignant, la conception de la leçon, la communication et l'évaluation des élèves. Ainsi, en France comme en Tunisie, cette conception de grilles d'évaluation renforce la légitimité de l'inspecteur d'EPS grâce à I'utilisation d'outils techniques (référentiel de compétences, grille d'observation, etc.), ce qui n'est pas sans rappeler le paradigme techniciste de Sénore (1999) qui donne une grande importance aux repères d'évaluation et qui se rapproche de la posture de contrôleur évoquée par Jorro (2006).

Trois inspecteurs français ne construisent pas de grilles d'évaluation puisque, selon eux, cela nécessiterait un travail collectif difficile à mener entre les IA-IPR d'une même académie. En Tunisie, 26 inspecteurs (33\%) ne se servent pas de grilles. Selon eux, la diversité des 


\section{eJRIEPS 48 Janvier 2021}

enseignants ainsi que la non-exhaustivité de la grille est la cause. Certains ajoutent qu'ils sont des experts et qu'ils se réfèrent à leur expérience et à leur " œil de maquignon » et n'ont pas besoin de grilles. Jorro (2006) considère que cette conception donne à l'inspecteur une posture d'expert qui maitrise le savoir ou une posture de juge puisqu'il cherche des adaptations et des aménagements. Sénore (2000) place cette conception dans le paradigme charismatique qui est le fondateur de l'acte d'inspection. Selon ce paradigme l'inspecteur est un contrôleur qui laisse de la place à l'arbitraire.

Ainsi, une majorité des inspecteurs plus importante en France qu'en Tunisie construit des grilles d'évaluation dans le but d'évaluer par rapport à une norme plus ou moins implicite et dans la recherche d'une objectivité.

\subsection{Question 4 : «pensez-vous qu'il soit important de clarifier à l'enseignant les} critères et les indicateurs d'évaluation avant l'inspection ? Quelle que soit votre réponse, argumentez. 》

Du fait de la différence institutionnelle entre les deux pays en matière de repères et de norme d'évaluation, il nous parait intéressant de savoir comment les inspecteurs déclarent interagir avec les enseignants à propos de l'évaluation. II s'agit encore d'une question fermée qui ramène à deux types de réponse, OUI ou NON. Aucun inspecteur n'a répondu « autres».

Les arguments de la réponse OUI mentionnés par les inspecteurs français et tunisiens s'organisent autour de deux catégories : «transparence » et " échange constructif ». Pour les arguments de la réponse NON, nous avons identifié à posteriori deux catégories : « les enseignants sont supposés savoir » et « il ne faut pas tout dire ». 


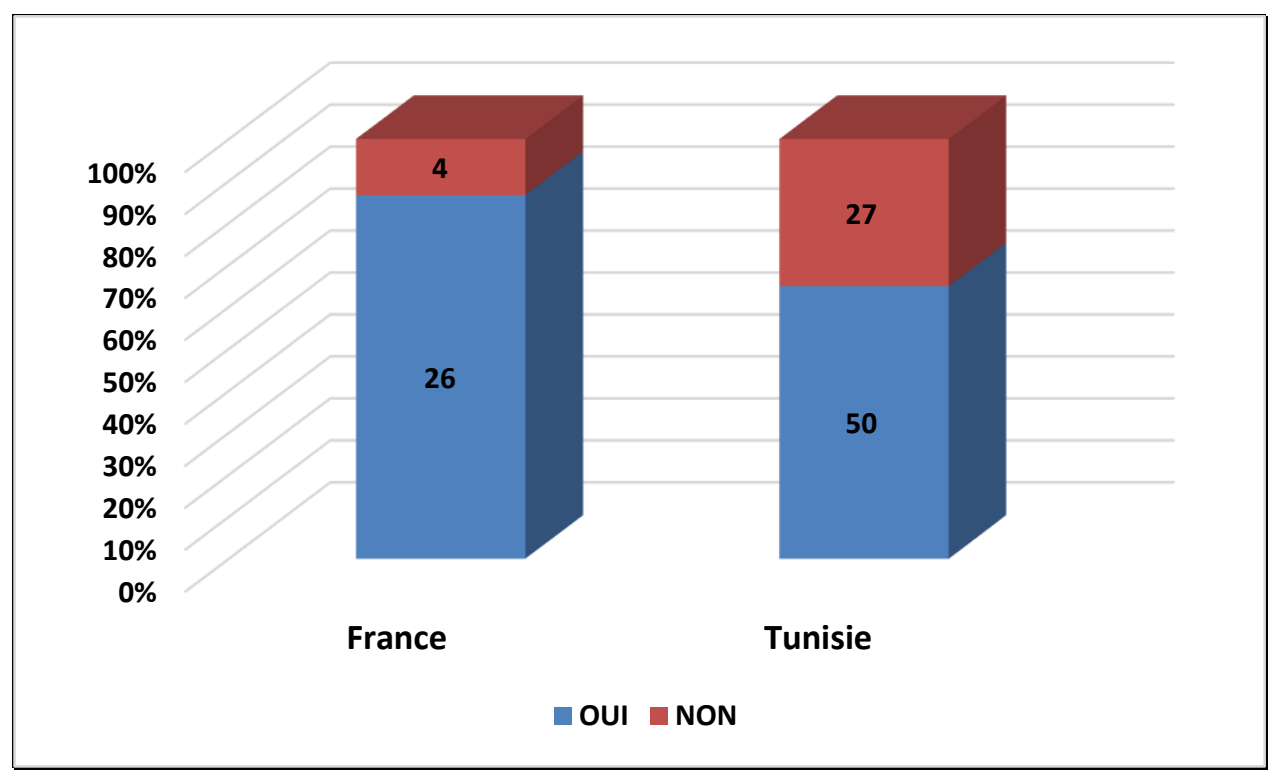

Figure 4: distribution des réponses à la question 4

Pour ce qui concerne cette question, 26 inspecteurs (86\%) français parmi ceux qui ont répondu au questionnaire pensent qu'il est important de clarifier à l'enseignant les critères et les indicateurs d'évaluation avant l'inspection. Ils précisent qu'ils annoncent ces critères dans la recherche d'une transparence et/ou dans le but d'instaurer un échange constructif autour des repères d'évaluation. En Tunisie 50 inspecteurs (65\%) vont dans le même sens en évoquant des arguments identiques. Cette conception renvoie à la posture du conseiller qui est à l'écoute et qui considère l'enseignant comme acteur (Jorro, 2006) et qui se rapproche du paradigme déontologique que nous avons évoqué en début d'article (Sénore, 1999) et dans lequel l'un des rôles les plus importants que peut jouer l'inspecteur est d'aider l'enseignant à devenir réellement un acteur pédagogique.

Quatre inspecteurs français pensent qu'il ne faut pas clarifier aux enseignants les critères et les indicateurs puisque ces derniers figurent dans le référentiel de compétences. En Tunisie, 27 inspecteurs parmi les participants pensent qu'il n'est pas important de clarifier à l'enseignant les indicateurs puisqu'ils sont supposés connus de tous. Cette conception se rapproche du paradigme charismatique de l'inspection qui n'a pas besoin de se justifier car il possède le savoir évaluatif, met à l'écart l'enseignant et maîtrise le contrôle de sa pratique. Ainsi, la majorité des inspecteurs pensent qu'il est important de clarifier les critères ou indicateurs de l'évaluation aux enseignants et évoquent les mêmes arguments dans les deux pays. Ils cherchent une certaine «transparence » de leur évaluation. En Tunisie, il semble persister une forme de résistance des inspecteurs pour divulguer et communiquer leurs critères d'évaluation. 


\section{eJRIEPS 48 Janvier 2021}

\subsection{Question 5 : «voyez-vous autrement la place de l'inspecteur dans le système éducatif ? Argumentez votre réponse. »}

Dans le but d'inciter les inspecteurs à lever le voile sur leur déjà-là conceptuel, il nous est apparu pertinent de savoir s'ils aimeraient changer leur place, leurs missions et leurs fonctions au sein de l'institution. Cette question fermée amène à deux types de réponse, OUI ou NON. Elle invite à argumenter quelle que soit la réponse.

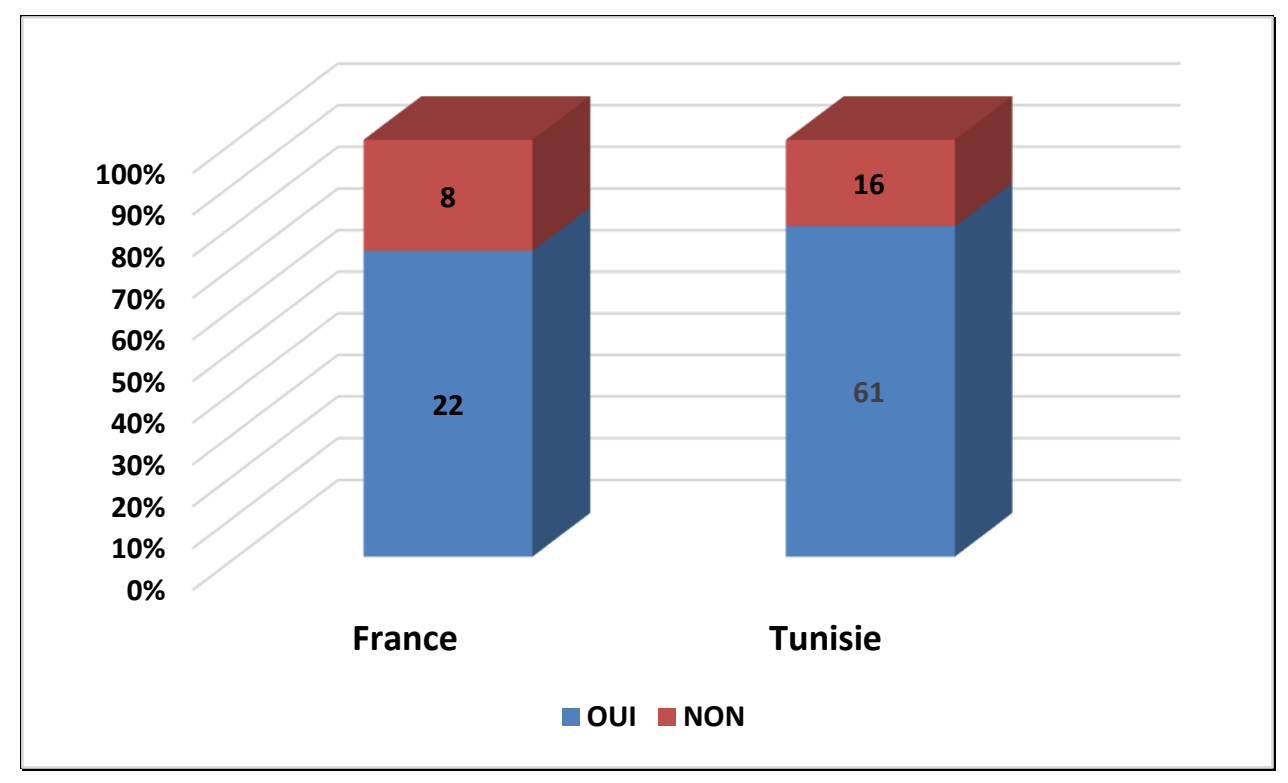

Figure 5: distribution des réponses à la question 5

En France, 22 inspecteurs (75\%) parmi les participants à la recherche voient autrement la place de l'inspecteur dans le système éducatif français. Malgré la mise en place du PPCR, ils continuent à penser que son rôle doit consister à accompagner et non à contrôler dans le but de former les enseignants. Certains utilisent la métaphore de l'« incubateur » pour montrer l'importance du rôle d'inspecteur dans le développement et l'avancement professionnel des enseignants ainsi que la participation dans les réformes de ce métier. 61 inspecteurs tunisiens $(79,3 \%)$ parmi ceux qui ont participé à cette recherche pensent également autrement la place de l'inspecteur dans leur système éducatif. Ils précisent que son rôle doit être plutôt tourné vers l'accompagnement et/ou l'élaboration des programmes d'EPS. Cette conception nous rappelle la posture de conseiller (Jorro, 2006) qui accompagne et qui considère l'enseignant comme acteur. Elle rejoint aussi la posture de l'expert, que l'inspecteur peut adopter, où il joue le rôle du coach qui favorise la progression et cherche l'efficacité de l'action.

II est intéressant de noter que seulement 8 inspecteurs ayant participé en France et 16 des inspecteurs ayant participé en Tunisie se disent satisfait de la place, des missions et des 


\section{eJRIEPS 48 Janvier 2021}

fonctions actuelles des inspecteurs dans le système éducatif et en conséquence ne souhaitent pas en changer. Ainsi, quel que soit le pays et malgré les différences interculturelles, plus des trois quarts des inspecteurs manifestent un désir d'être à une autre place dans le système éducatif. Cette insatisfaction au regard de l'institution est de nature à générer de l'inconfort voire un certain mal être. L'analyse didactique clinique des pratiques effectives d'inspection nous permettra peut-être de rendre compte de cette frustration et des effets qu'elle produit lors de l'épreuve interactive que ce soit l'observation de la leçon ou l'entretien qui suit.

\subsection{Question 6 : “ d'après vous à quoi sert l'entretien après l'observation? »}

Pour mettre en relief les conceptions dominantes relatives à l'intérêt de faire un entretien après l'observation lors du processus d'inspection, nous avons découpé les réponses à cette question ouverte en unités de sens et les avons regroupées à posteriori en quatre catégories: comprendre (bilan, préciser/expliciter ses intentions et/ou ses décisions), pratique réflexive (échange, prise de distance), perspectives (pistes et conseils pour optimiser la pratique), évaluer des connaissances (niveau de formation). Le graphique 6 représente la distribution en pourcentage des conceptions dominantes relatives à l'intérêt et au sens que les inspecteurs donnent à l'entretien qui suit l'observation de la leçon. Nous ne traitons ici que les réponses placées en premier ordre et qui indiquent la conception dominante.

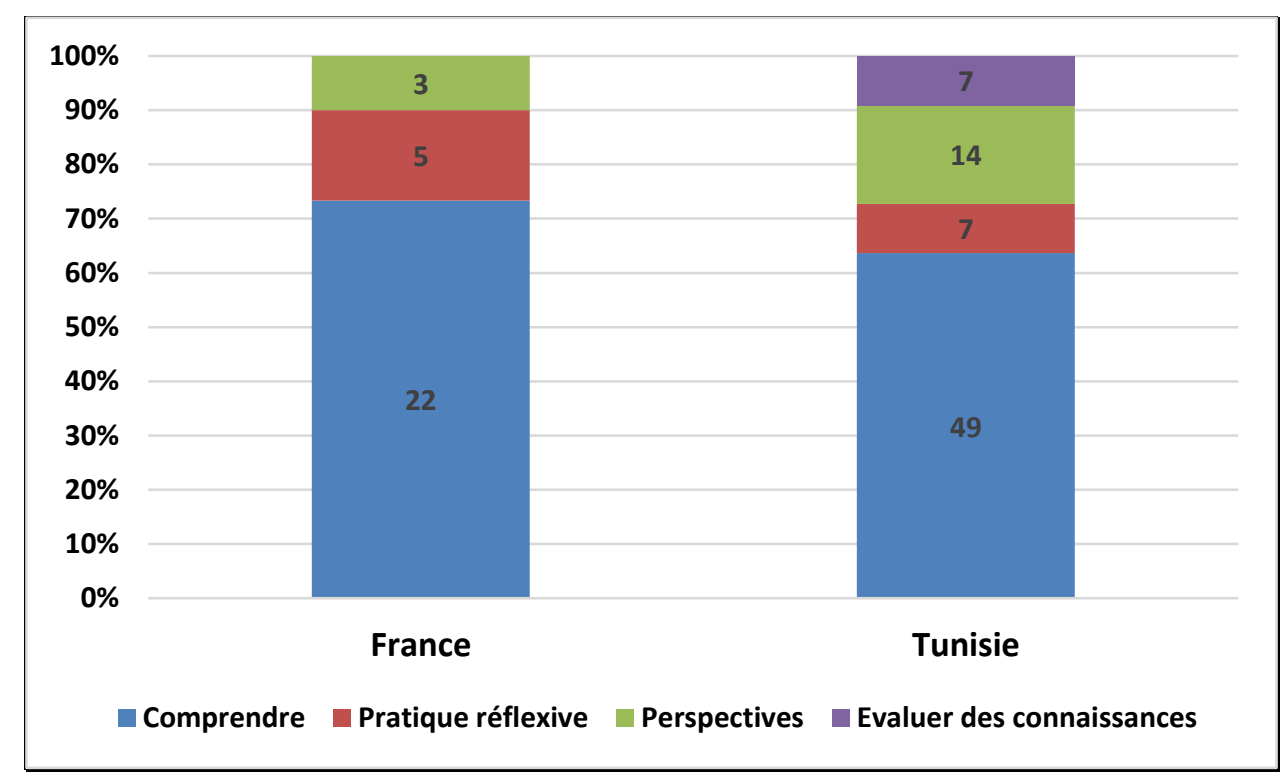

Figure 6: distribution des réponses à la question 6 


\section{eJRIEPS 48 Janvier 2021}

Du côté des français, plus de $71 \%$ des inspecteurs pensent prioritairement que l'entretien est un moment qui permet à l'enseignant d'expliciter ses intentions et ses décisions. II offre en même temps l'occasion à l'enseignant et à l'inspecteur d'élaborer un bilan de nature à échanger sur les choix didactiques et pédagogiques afin de mieux les comprendre. Près de $18 \%$ d'entre eux pensent en premier lieu qu'à travers l'entretien, ils peuvent évaluer la capacité de l'enseignant à réfléchir sur sa pratique (pratique réflexive). Près de $11 \%$ des réponses mettent en avant le rôle premier de l'entretien dans la recherche de pistes et de perspectives pour optimiser la pratique.

Au-delà des différences culturelles, en Tunisie, comme en France, la majorité des inspecteurs (64\%) ayant répondu considèrent prioritairement l'entretien comme un moment privilégié pour mieux comprendre le travail de l'enseignant. Environ 9\% des inspecteurs tunisiens et $18 \%$ des français pensent en premier que l'entretien met en relief la capacité de l'enseignant à développer une pratique réflexive. $17 \%$ des tunisiens et près de $11 \%$ des français mettent en avant le rôle important de l'entretien dans la recherche de pistes et de perspectives de développement professionnel. Environ 10\% des inspecteurs tunisiens pensent que ce moment d'inspection leur permet d'évaluer les connaissances didactiques ou pédagogiques de l'enseignant, cette réponse n'apparaît jamais du côté des français. Ainsi quelles que soient les directives institutionnelles, la conception dominante de plus de la majorité des inspecteurs d'EPS tunisiens et français concernant l'intérêt de l'entretien après l'observation de la leçon semble se construire autour d'une meilleure compréhension du processus décisionnel de l'enseignant. Cette conception fait écho à la posture compréhensive évoquée par Jorro (2006) où l'inspecteur essaye de décrypter la situation afin d'apporter des régulations adéquates. Elle est également en phase avec le paradigme déontologique proposé par Sénore (1999). La deuxième conception dominante des inspecteurs français, relative à cette question, pointe l'intérêt de repérer ou de susciter la capacité des enseignants à développer une réflexivité sur sa pratique. En fonction des intentions de l'inspecteur, cette conception renvoie soit à la posture du contrôleur soit à la posture de l'accompagnateur (Jorro, 2006). En Tunisie, la deuxième conception dominante sur l'utilité de l'entretien renvoie à l'élaboration de perspectives de progression. Cette conception nous rappelle la posture d'expert de Jorro (2006) qui favorise la progression professionnelle. 


\section{eJRIEPS 48 Janvier 2021}

\subsection{Question 7 : quels sont les qualificatifs que vous utiliseriez pour définir le profil d'un enseignant d'EPS compétent ?}

Pour cette dernière question ouverte nous avons choisi de ne traiter que les unités de sens qui arrivent en premier dans les réponses des inspecteurs de manière à rendre compte de leurs conceptions dominantes de l'enseignant d'EPS compétent. La lecture de ces premiers items s'est faite au filtre du référentiel de compétences communes à tous les professeurs et personnels d'éducation en France (B.O. du 25 juillet 2013) qui liste 14 compétences communes à tous les professeurs et personnels d'éducation (CCPPE) ainsi que 5 compétences communes à tous les professeurs (CCP) qu'ils doivent maîtriser pour l'exercice de leur métier (annexes 2). Pour rappel, il n'existe pas en Tunisie un référentiel métier officiel pour les enseignants. II nous paraît donc intéressant de repérer si du côté des français les IA IPR EPS se réfèrent explicitement ou implicitement au référentiel de 2013 et si du côté tunisien on retrouve certains éléments de ce référentiel. $A$ travers ce filtre d'analyse nous avons identifié et construit a posteriori six catégories mettant en avant des qualificatifs dominants ciblant une ou plusieurs compétences du référentiel :

1. Praticien «éthique et responsable » regroupant des qualificatifs d'ordre général relatifs à la posture de l'enseignant et ciblant particulièrement les CCPPE 1, 2 et 3 (annexes 2).

2. Praticien intégré dans un collectif et doté d'un « esprit d'équipe » à l'échelle disciplinaire, interdisciplinaire et inter catégorielle. Cette catégorie regroupe les CCP 1 et 2 (annexes 2).

3. Praticien maîtrisant les « savoirs disciplinaires/ APSA » qui équivaut à la CCP 1 du référentiel (annexes 2).

4. Praticien « réflexif » soucieux de faire évoluer ses pratiques notamment par la formation continue. Cette catégorie renvoie à la CCPPE 14 (annexes 2).

5. Praticien « soucieux de la réussite des élèves » regroupant des notions centrées sur l'élève (éducabilité, réussite, individualisation, différentiation, etc.) et renvoyant aux CCPPE 3, 4 et 5 ainsi que la CCP 3 du référentiel (annexes 2).

6. La cinquième catégorie intitulée « autres compétences » rassemble toutes les autres réponses. Ces dernières sont relatives à la maîtrise de savoirs professionnels évoqués dans les compétences suivantes : CCPPE 7, CPE 2, 3 et 5 (annexes 2). 


\section{eJRIEPS 48 Janvier 2021}

Le graphique 7 représente la distribution en nombre et en pourcentage des conceptions dominantes relative à l'enseignant d'EPS compétent chez les inspecteurs français et tunisiens.

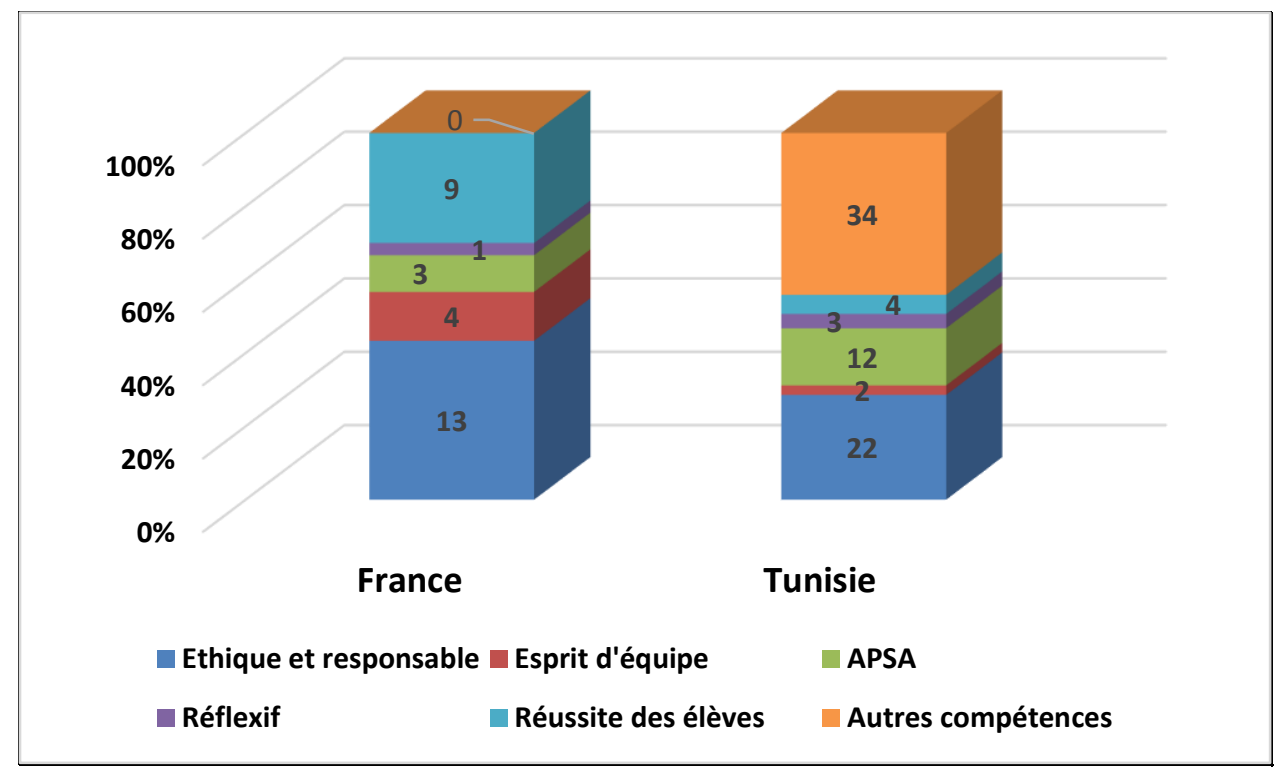

Figure 7 : distribution des réponses à la question 7

Sur les 19 compétences que compte le référentiel, les regroupements de réponses que nous avons opérés ciblent directement ou indirectement 15 d'entre-elles. Les 4 compétences qui ne sont jamais évoquées dans le premier item des réponses des inspecteurs français comme tunisiens sont :

- CCPPE 8 : Utiliser une langue vivante étrangère dans les situations exigées par son métier

- CCPPE 9 : Intégrer les éléments de la culture numérique nécessaires à l'exercice de son métier

- CCPPE 12 : Coopérer avec les parents d'élèves

- CCPPE 13 : Coopérer avec les partenaires de l'école

Ainsi, ces 4 compétences sont absentes ou jugées secondaires par les inspecteurs d'EPS pour qualifier un enseignant compétent.

Du côté des français, $42 \%$ des inspecteurs ( $n=13$ ) pensent prioritairement qu'un enseignant compétent est d'abord un praticien éthique et responsable qui fait partager les valeurs de la République (CCPPE1), qui s'inscrit dans le cadre des principes fondamentaux du système éducatif (CCPPE2) et agit comme un éducateur responsable et selon des principes éthiques (CCPPE6). Avec un score de $28 \%(n=22)$, cette réponse arrive en deuxième chez les inspecteurs tunisiens pour qui la conception dominante pour près d'un inspecteur sur deux 


\section{eJRIEPS 48 Janvier 2021}

$(n=34)$ renvoie à la maîtrise de certains savoirs professionnels (concevoir, conduire, animer, communiquer, évaluer, réguler) qui sont au cœur de 3 des 5 CCP et d'une CCPPE du référentiel français (voir annexes). Si cette catégorie apparaît çà et là dans les réponses des inspecteurs français, elle n'apparaît jamais en premier item dans les réponses des 30 inspecteurs français. Evidence, allant de soi ou " effet référentiel explicite ", nous relevons là, une différence culturelle importante qu'il nous est difficile de comprendre et encore moins d'interpréter à ce stade de l'étude entre le déjà-là conceptuel des inspecteurs français et celui de leurs homologues tunisiens. II sera intéressant d'aller voir lors des études de cas quelles sont les préoccupations premières des 4 sujets qui ont accepté de collaborer à notre recherche.

La deuxième catégorie que l'on trouve chez $30 \%$ des IA IPR EPS français $(n=9)$ met en relief qu'un enseignant compétent est un praticien qui connait ses élèves, prend en compte leur diversité afin de les accompagner dans leur réussite scolaire et sociale. Cette conception d'un praticien soucieux de la réussite des élèves renvoie à 4 compétences du référentiel (CCPPE 3, 5 et 5 et CCP 4). Cette centration au service de la réussite des élèves ne se retrouve que dans $5 \%$ des réponses des inspecteurs tunisiens $(n=4)$.

Pour près de $13 \%$ des répondants français $(n=4)$ et $3 \%$ des tunisiens $(n=2)$, on trouve une conception dominante mettant l'accent sur le sens collectif, le travail et l'esprit d'équipe ainsi que la collaboration avec l'ensemble des membres de la communauté éducative (CCPPE 10 et 11). Cette conception dominante arrive en $3^{\text {ème }}$ position chez les français alors qu'elle est en $6^{\text {ème }}$ et dernière position chez les tunisiens (tableau IV des annexes).

La centration sur la spécificité disciplinaire à travers la connaissance des APSA et leur traitement didactique en EPS apparaît en troisième position des conceptions dominantes chez les inspecteurs tunisiens et en avant-dernière position chez les inspecteurs français. En effet, pour $16 \%$ des tunisiens $(n=12)$ et $10 \%$ des français $(n=3)$ un enseignant d'EPS compétent est d'abord un praticien qui maîtrise les savoirs disciplinaires et la didactique des APSA (APS pour les tunisiens) inscrites dans les programmes d'EPS. Ce constat amène à penser que pour une grande majorité d'IPR d'EPS, un enseignant d'EPS est d'abord un enseignant, un agent du service public d'éducation et membre d'une communauté éducative avant d'être un spécialiste des apprentissages moteurs. Ce constat est de nature à questionner l'évolution de la discipline EPS au sein du système éducatif, en particulier en France, où au cours de ces quatre dernières décennies la conception culturaliste de la discipline semble s'être affaiblie au profit d'une conception intégrative, que la mise en œuvre 


\section{eJRIEPS 48 Janvier 2021}

du socle commun de connaissances et de compétences a certainement favorisée et précipitée.

La dernière conception dominante de l'enseignant d'EPS compétent qui apparaît à travers les réponses des inspecteurs français à cette ultime question est celle du praticien réflexif. Un praticien qui par divers moyens (analyse de pratique, formation continue, échanges collectifs) s'engage dans une démarche individuelle et collective de développement professionnel comme le mentionne la CCPPE 14 du référentiel français. Pour 3 IA IPR EPS français (4\%) et 3 tunisiens (également $4 \%$ ) cette catégorie arrive en première position dans les énoncés des réponses à cette question. La réflexivité renvoie à la capacité de réfléchir délibérément sur ses propres pratiques (Perrenoud, 2001) en vue de les améliorer (Tochon, 1993 ; Schön, 1996). Ces auteurs ont fortement marqué la formation des enseignants en particulier en EPS au cours de ces trente dernières années. Le faible score obtenu par cette catégorie masque le fait que si ce qualificatif de « réflexif » n'arrive que rarement en premier item, il est très présent dans le corpus global des réponses. Le traitement de cette question pour lequel nous avons opté et qui consiste à ne regarder que les premiers qualificatifs énoncés par les répondants mériterait d'être affiné et nuancé au regard d'une analyse plus globale des réponses. Notre intention étant ici de n'analyser que les conceptions dominantes donc a priori celles qui arrivent en premier dans les réponses des inspecteurs. Ainsi, l'on constate qu'en l'absence d'un référentiel de compétences des enseignants en Tunisie, la plupart des compétences existantes dans le référentiel français sont présentes dans le déjà-là conceptuel des inspecteurs, ce qui en soi n'est pas surprenant: un enseignant d'EPS français comme tunisien est d'abord un enseignant et poursuit la même finalité du service public d'éducation à savoir « former un citoyen cultivé, autonome, lucide et responsable ». Par ailleurs, en France, il semblerait que la publication officielle d'un référentiel de compétences ne se substitue pas au référent implicite de chacun en matière de ce qu'est ou devrait être un enseignant d'EPS compétent. Au-delà de la singularité des sujets interrogés et de leurs réponses, nous mettons en avant des différences et des régularités interculturelles en lien avec les injonctions de chaque institution que la suite qualitative de nos travaux et analyses ne manquera pas d'interroger et de préciser.

\section{Conclusion}

Dans les limites de validité locale et contextuelle (Van Der Maren, 1995), ce questionnaire exploratoire revêt pour notre étude un double intérêt. D'une part il fait émerger certains 


\section{eJRIEPS 48 Janvier 2021}

éléments saillants et génériques d'un déjà-là conceptuel, en tension entre contrôle et accompagnement, chez un nombre relativement important d'inspecteurs d'EPS tunisiens et français quelle que soit leur appartenance institutionnelle. D'autre part, en prévision des études de cas qui nourriront notre recherche, ce questionnaire nous permet de positionner les quatre inspecteurs qui ont accepté de collaborer à cette étude. Cet article s'est focalisé sur le premier aspect et met en évidence la multiplicité et la complexité des réseaux conceptuels des inspecteurs d'EPS plus ou moins assujettis à leurs institutions de rattachement.

Entre sphère publique et privée, influences culturelles, institutionnelles et personnelles, les conceptions des inspecteurs relatives à leur difficile et délicate mission évaluative en EPS s'élaborent et émergent selon une logique singulière difficilement accessible et culturellement dépendante. Si comme l'affirme Jorro (2009), « la dimension culturelle importe au plus haut point puisqu'elle joue un rôle de configuration de l'action évaluative » (Jorro, 2009, p 219), la part du sujet inspecteur, notamment dans son rapport à l'institution, apparaît comme un élément incontournable dans la compréhension du processus d'inspection. Entre accompagnement et contrôle, ces analyses révèlent et corroborent un certain nombre de tensions voire de contradictions au sein d'un déjà-là conceptuel agissant de manière constante et latente en amont de la décision (Carnus, 2002) comme filtre de l'action didactique (Loizon, 2004).

A travers ces modestes résultats, et au-delà des différences culturelles, nous pointons chez les inspecteurs d'EPS en Tunisie et en France une variabilité inter et intra individuelle dans l'adoption de différentes postures d'évaluation (Jorro, 2006) comme celles de contrôleur, de juge, d'expert, de conseiller en lien avec différents paradigmes : charismatique, techniciste et déontologique (Sénore, 1999). Ce constat corrobore certains travaux selon lesquels « l'évaluateur circule entre les postures et les paradigmes » dans une même séance (Jorro, 2009, p 73).

Ainsi, en prenant en compte le sujet dans ses dimensions psychologique et sociale (Beillerot, Blanchard-Laville, Mosconi, 1996), l'approche clinique en didactique est de nature à apporter une autre intelligibilité à la complexité des conceptions des inspecteurs. Notre recherche se poursuit selon la méthodologie didactique clinique. Des entretiens avec nos quatre collaborateurs inspecteurs permettront de dessiner plus précisément les contours de leur déjà-là conceptuel singulier et de repérer avec une plus grande acuité les tensions structurelles qui les animent avant d'aller les observer, in situ et au cas par cas au cours de visites d'inspection. Qu'en sera-t-il des tensions qui les animent et comment - au cas par 


\section{eJRIEPS 48 Janvier 2021}

cas - les gèreront-ils ? Entre accompagnement et contrôle, quels compromis concèderontils ? Quels seront les savoirs réellement évalués ? Et quelles seront les traces de leurs déjàlà conceptuel au moment de l'épreuve de l'inspection?

\section{Bibliographie}

Albanel, X. (2009). Le travail d'évaluation. L'inspection dans l'enseignement secondaire. Toulouse : Octarès.

Aroui, M.-S. (2021). Analyse didactique clinique de pratiques d'inspection en EPS. Etude de cas croisés en France et en Tunisie. Thèse de doctorat en Sciences de l'Education et de la Formation. Université Paul Sabatier, Toulouse.

Beillerot, J., Blanchard-Laville, C., \& Mosconi, N. (1996). Pour une clinique du rapport au savoir. Paris: L'Harmattan.

Carnus, M.-F. (2001). Analyse didactique du processus décisionnel de l'enseignant d'EPS en gymnastique. Une étude de cas croisés. Toulouse: Thèse de doctorat en sciences de l'éducation publiée aux presses universitaires européenne en 2017.

Carnus, M.-F. (2002). Croyances, conceptions, intentions et pratiques effectives dans l'enseignement de la gymnastique : Le cas de l'ATR et du repérage. In J.-F. Robin et A. Durny (dir), Travaux d'actualité en activités gymniques et acrobatiques (p. 160164). Dossiers EPS, 57, Paris.

De Ketele, J.-M., \& Roegiers, X. (2016). Méthodologie du recueil d'informations. Bruxelles: De Boeck Université.

Décret gouvernemental $n^{\circ}$ 2019-920 du 26 septembre 2019, portant statut particulier des membres du corps de l'inspection pédagogique du ministère des affaires de la jeunesse et des sports.

Figari, G. \& Remaud, D. (2014). Chapitre 6. La place des référentiels dans l'évaluation. Dans C. Tourmen, Méthodologie d'évaluation en éducation et formation: Ou l'enquête évaluative (pp. 65-69). Louvain-la-Neuve : De Boeck Supérieur.

Giordan A. et de Vecchi G. (1987). Les origines du savoir, des conceptions des apprenants aux concepts scientifiques. Paris : Delachaux et Niestlé.

Hébrard, A. (1986) . L'éducation Physique et Sportive, réflexions et perspectives. Paris : Revue EPS.

Jorro, A. (2006). L'ethos de l'évaluateur: entre imaginaires et postures. Dans G. Figari \& L. Mottier Lopez (éds), Recherche sur l'évaluation en éducation (pp. 68-75). Paris : L'Harmattan. 


\section{eJRIEPS 48 Janvier 2021}

Jorro, A. (2009). L'évaluation comme savoir professionnel. Dans L. Mottier Lopez \& M. Crahay (éds), Evaluations en tension (pp. 219-231). Paris: De Boeck.

Loizon, D. (2004). Analyse des pratiques d'enseignement du judo : identification du savoir transmis à travers les variables didactiques utilisées par les enseignants en club et en EPS. Thèse de doctorat non publiée, Université Paul Sabatier, Toulouse.

Loizon, D. \& Carnus M.-F. (2012). Le déjà-là décisionnel chez les enseignants novices en EPS. Un outil pour comprendre la réalité de leurs enseignements, in Carlier (G.), Borges (C.), Clerx (M.) et Delens (C.), Identité professionnelle en éducation physique. Parcours des stagiaires et enseignants novices (pp. 199-215). Louvainla-Neuve : Presses Universitaires de Louvain,

Loizon, D. \& Carnus M.-F. (2012). Le déjà-là décisionnel chez les enseignants novices en EPS. Un outil pour comprendre la réalité de leurs enseignements, in G. Carlier, C. Borges, M. Clerx et C. Delens, Identité professionnelle en éducation physique. Parcours des stagiaires et enseignants novices (pp.199-215). Louvain-la-Neuve : Presses Universitaires de Louvain.

Paquay, L., Wouters, P. \& Van Nieuwenhoven, C. (2010). Introduction. L'évaluation, frein ou levier du développement professionnel ? Dans L. Paquay (Ed.), L'évaluation, levier du développement professionnel : Tensions, dispositifs, perspectives (pp. 834). Louvain-la-Neuve : De Boeck Supérieur.

Perrenoud, P. (2001). Développer la pratique réflexive dans le métier d'enseignant. Professionnalisation et raison pédagogique. Paris : ESF.

Saint-Arnaud, Y. (2001). La réflexion dans l'action, un changement de paradigme. Recherche et formation, 36, 17-27.

Sénore, D. (1999). L'inspecteur, le maitre et les élèves. Lyon : thèse de doctorat en sciences de l'éducation non publiée.

Sénore, D. (2000). Pour une éthique de l'inspection. Issy-les-Moulineaux : ESF.

Schön, D.A. (1996). The reflexive practitioner. How professionals think in action. Aldershot : Arena.

Schön, D.A. \& Argyris, C. (1999). Théorie et pratique professionnelle. Comment en accroître l'efficacité. Québec : Logiques.

Tochon, F.V. (1993). L'enseignant expert. Paris : Nathan.

Vacher, J. (1986). L'inspecteur et son image, L. GILLIG AMOROS. Paris : PUF. 
eJRIEPS 48 Janvier 2021

Van Der Maren, J. M. (1995). Méthodes de recherche pour l'éducation. Bruxelles : De Boeck Université.

\section{Webographie}

Inspecteur d'académie. Inspecteur Pédagogique Régional (M.E.N.J.S.)

https://www.education.gouv.fr/inspecteur-d-academie-inspecteur-pedagogiqueregional-ia-ipr-2666

Mutations et réformes de structures de l'enseignement en Tunisie : http://aan.mmsh.univaix.fr/volumes/1967/Pages/AAN-1967-06 36.aspx

Référentiel des compétences des enseignants et des conseillers principaux d'éducation. Bulletin officiel du 25 juillet 2013 : https://www.education.gouv.fr/le-referentiel-decompetences-des-metiers-du-professorat-et-de-l-education-5753 


\section{Annexes 1 : L'inspection en EPS en Tunisie et en France : éléments de comparaison}

Tableau I: comparatif des parcours de formation et des missions des inspecteurs en Tunisie et en France

\begin{tabular}{|c|c|c|}
\hline & Tunisie & France \\
\hline $\begin{array}{l}\text { Institution de } \\
\text { tutelle }\end{array}$ & $\begin{array}{l}\text { - Administration centrale du } \\
\text { ministère (MAJS) } \\
\text { - Commissariat régional } \\
\text { - Circonscription }\end{array}$ & $\begin{array}{l}\text { - Académie (recteur) } \\
\text { - Ministère de l'éducation } \\
\text { nationale (MEN) }\end{array}$ \\
\hline Recrutement & $\begin{array}{l}\text { - Concours puis formation pendant } \\
24 \text { mois }\end{array}$ & $\begin{array}{l}\text { - Concours, soit listes d'aptitude } \\
\text { ou détachement. Formation } 24 \\
\text { mois en deux périodes de } 12 \\
\text { mois. (Avant et après la } \\
\text { titularisation) }\end{array}$ \\
\hline $\begin{array}{l}\text { Contenu des } \\
\text { épreuves du } \\
\text { concours }\end{array}$ & $\begin{array}{l}\text { - Épreuve écrite d'admissibilité } \\
\text { (didactique des APS, pédagogie de } \\
\text { l'EPS) } \\
\text { - Épreuve pratique d'admission : } \\
\text { enseigner une séance d'EPS+ } \\
\text { entretien avec le jury }\end{array}$ & $\begin{array}{l}\text { - Épreuve d'admissibilité : sur } \\
\text { dossier de reconnaissance des } \\
\text { acquis de l'expérience } \\
\text { professionnelle noté de } 0 \text { à } 20 \\
\text { (double correction). } \\
\text { - Épreuve orale d'admission notée } \\
\text { de } 0 \text { à } 20 \\
\text { - Exposé sur le parcours et } \\
\text { l'activité professionnelle } \\
\text { - Entretien ( } 45 \text { min) avec un } \\
\text { jury }\end{array}$ \\
\hline $\begin{array}{l}\text { Contenu de } \\
\text { la formation } \\
\text { et durée }\end{array}$ & $\begin{array}{l}\text { - Théorique : didactique des APS, } \\
\text { législation } \\
\text { - Pratique : encadrée par un } \\
\text { inspecteur } \\
24 \text { mois }\end{array}$ & $\begin{array}{l}\text { - Exercice effectif des fonctions } \\
\text { d'inspecteur } \\
\text { - Des sessions de formations à } \\
\text { I'IH2EF de Poitiers } \\
24 \text { mois }\end{array}$ \\
\hline $\begin{array}{l}\text { Validation de } \\
\text { la formation }\end{array}$ & $\begin{array}{l}\text { - Épreuve écrite sur la formation } \\
\text { théorique en contrôle continu. } \\
\text { - Épreuve pratique : inspecter une } \\
\text { séance } \\
\text { - Présentation d'un projet de } \\
\text { recherche }\end{array}$ & $\begin{array}{l}\text { - Avis de la commission } \\
\text { administrative paritaire nationale } \\
\text { - Un rapport établi par le directeur } \\
\text { du centre de formation } \\
\text { - Un rapport de stage établi par le } \\
\text { Recteur d'Académie concerné }\end{array}$ \\
\hline Titularisation & $\begin{array}{l}\text { Avis favorable du directeur national } \\
\text { de l'inspection pédagogique. }\end{array}$ & $\begin{array}{l}\text { Rapport établi par le doyen de } \\
\text { l'inspection générale de } \\
\text { l'éducation nationale (IGEN) }\end{array}$ \\
\hline Missions & $\begin{array}{l}\text { Évaluation, formation et innovation, } \\
\text { contrôle, accompagnement }\end{array}$ & $\begin{array}{l}\text { Évaluation, formation, animation } \\
\text { pédagogique, accompagnement, } \\
\text { contrôle }\end{array}$ \\
\hline
\end{tabular}


Tableau II : les principaux éléments du processus d'inspection dans les deux pays

\begin{tabular}{|l|l|l|}
\hline & Tunisie & France \\
\hline $\begin{array}{l}\text { Evaluation du } \\
\text { travail des } \\
\text { enseignants }\end{array}$ & $\begin{array}{l}\text { Inspection individuelle à } \\
\text { l'improviste : } \\
- \text { Contact avec le proviseur } \\
- \text { Observation de la leçon } \\
\text { - Entretien en présence du } \\
\text { proviseur } \\
\text { - Envoi du rapport d'inspection à } \\
\text { l'administration centrale } \\
\text { d'inspection qui l'envoie à } \\
\text { l'enseignant }\end{array}$ & $\begin{array}{l}\text { Inspection individuelle ou } \\
\text { collective, annoncée 15 jours } \\
\text { avant : } \\
- \text { Contact avec l'enseignant et } \\
\text { aussi le proviseur } \\
- \text { Observation de la leçon } \\
- \text { Entretien } \\
- \text { Rapport par notification et par } \\
\text { mail }\end{array}$ \\
\hline $\begin{array}{l}\text { Référentiel de } \\
\text { compétences }\end{array}$ & Non & $\begin{array}{l}\text { Oui } \\
\text { Bulletin officiel du 25 juillet 2013. }\end{array}$ \\
\hline $\begin{array}{l}\text { Décision } \\
\text { Evaluation }\end{array}$ & Note & Appréciation \\
\hline
\end{tabular}

\section{Annexes 2 : Eléments d'analyse de la question 7}

Référentiel des compétences des enseignants et des conseillers principaux d'éducation publiée au bulletin officiel du 25 juillet 2013.

Compétences communes à tous les professeurs et personnels d'éducation (CCPPE)

- CCPPE 1 : Faire partager les valeurs de la République

- CCPPE 2 : Inscrire son action dans le cadre des principes fondamentaux du système éducatif et dans le cadre réglementaire de l'école

- CCPPE 3 : Connaître les élèves et les processus d'apprentissage

- CCPPE 4 : Prendre en compte la diversité des élèves

- CCPPE 5 : Accompagner les élèves dans leur parcours de formation

- CCPPE 6 : Agir en éducateur responsable et selon des principes éthiques

- CCPPE 7 : Maîtriser la langue française à des fins de communication

- CCPPE 8 : Utiliser une langue vivante étrangère dans les situations exigées par son métier

- CCPPE 9 : Intégrer les éléments de la culture numérique nécessaires à l'exercice de son métier

- CCPPE 10 : Coopérer au sein d'une équipe

- CCPPE 11 : Contribuer à l'action de la communauté éducative

- CCPPE 12 : Coopérer avec les parents d'élèves

- CCPPE 13 : Coopérer avec les partenaires de l'école

- CCPPE 14: S'engager dans une démarche individuelle et collective de développement professionnel

Compétences communes à tous les professeurs (CCP)

- CCP 1 : Maîtriser les savoirs disciplinaires et leur didactique

- CCP 2 : Maîtriser la langue française dans le cadre de son enseignement

- CCP 3 : Construire, mettre en œuvre et animer des situations d'enseignement et d'apprentissage prenant en compte la diversité des élèves 


\section{eJRIEPS 48 Janvier 2021}

- CCP 4 : Organiser et assurer un mode de fonctionnement du groupe favorisant l'apprentissage et la socialisation des élèves

- CCP 5 : Évaluer les progrès et les acquisitions des élèves

Tableau III : regroupement des réponses à la question 7

\begin{tabular}{|c|c|}
\hline Catégorie & Compétences du référentiel \\
\hline $\begin{array}{l}\text { Éthique et } \\
\text { responsable }\end{array}$ & $\begin{array}{l}\text { CCPPE } 1: \text { Faire partager les valeurs de la République } \\
\text { CCPPE } 2: \text { Inscrire son action dans le cadre des principes } \\
\text { fondamentaux du système éducatif et dans le cadre } \\
\text { réglementaire de l'école } \\
\text { CCPPE } 6 \text { : Agir en éducateur responsable et selon des principes } \\
\text { éthiques }\end{array}$ \\
\hline $\begin{array}{l}\text { Soucieux de la } \\
\text { réussite des } \\
\text { élèves }\end{array}$ & $\begin{array}{l}\text { CCPPE } 3 \text { : Connaître les élèves et les processus d'apprentissage } \\
\text { CCPPE } 4 \text { : Prendre en compte la diversité des élèves } \\
\text { CCPPE } 5 \text { : Accompagner les élèves dans leur parcours de } \\
\text { formation } \\
\text { CCP } 4 \text { : Organiser et assurer un mode de fonctionnement du } \\
\text { groupe favorisant l'apprentissage et la socialisation des élèves }\end{array}$ \\
\hline APSA & CCP 1 : Maîtriser les savoirs disciplinaires et leur didactique \\
\hline Esprit d'équipe & $\begin{array}{l}\text { CCPPE } 10 \text { : Coopérer au sein d'une équipe } \\
\text { CCPPE } 11: \text { Contribuer à l'action de la communauté éducative }\end{array}$ \\
\hline Réflexif & $\begin{array}{l}\text { CCPPE 14: S'engager dans une démarche individuelle et } \\
\text { collective de développement professionnel }\end{array}$ \\
\hline $\begin{array}{l}\text { Autres savoirs } \\
\text { professionnels }\end{array}$ & $\begin{array}{l}\text { CCPPE } 7 \text { : Maîtriser la langue française à des fins de } \\
\text { communication } \\
\text { CCP } 2 \text { : Maîtriser la langue française dans le cadre de son } \\
\text { enseignement } \\
\text { CCP } 3 \text { : Construire, mettre en œuvre et animer des situations } \\
\text { d'enseignement et d'apprentissage prenant en compte la diversité } \\
\text { des élèves } \\
\text { CCP } 5 \text { : Évaluer les progrès et les acquisitions des élèves }\end{array}$ \\
\hline
\end{tabular}

Tableau IV : Classement en pourcentage des conceptions dominantes chez les inspecteurs tunisiens et français à propos de l'enseignant d'EPS compétent.

\begin{tabular}{|c|l|l|}
\hline Conception & Inspecteurs tunisiens (en \%) & Inspecteurs français (en \%) \\
\hline $\mathbf{1}$ & $\begin{array}{l}\text { Autres savoirs professionnels } \\
(48 \%)\end{array}$ & $\begin{array}{l}\text { Ethique et responsable } \\
(42 \%)\end{array}$ \\
\hline $\mathbf{2}$ & Ethique et responsable (28\%) & Soucieux de la réussite des élèves (30\%) \\
\hline $\mathbf{3}$ & $\begin{array}{l}\text { Savoirs disciplinaires / APSA } \\
(16 \%)\end{array}$ & $\begin{array}{l}\text { Esprit d'équipe } \\
(13 \%)\end{array}$ \\
\hline $\mathbf{4}$ & $\begin{array}{l}\text { Soucieux de la réussite des } \\
\text { élèves (5\%) }\end{array}$ & $\begin{array}{l}\text { Savoirs disciplinaires / APSA } \\
(10 \%)\end{array}$ \\
\hline $\mathbf{5}$ & Réflexif (4\%) & Réflexif (4\%) \\
\hline $\mathbf{6}$ & Esprit d'équipe (3\%) & \\
\hline
\end{tabular}

